\title{
Quantification of white matter injury following neonatal stroke with serial DTI
}

Niek E. van der Aa' ${ }^{1}$ Frances J. Northington ${ }^{2}$, Brian S. Stone ${ }^{2,3}$, Floris Groenendaal' ${ }^{1}$, Manon J.N.L. Benders', Giorgio Porro ${ }^{4}$, Shoko Yoshida ${ }^{5,6}$, Susumu Mori ${ }^{5,6}$, Linda S. de Vries ${ }^{1}$ and Jiangyang Zhang ${ }^{5}$

BACKGROUND: Diffusion tensor imaging (DTI) can be used to predict outcome following perinatal arterial ischemic stroke (PAIS), although little is known about white matter changes over time.

METHODS: Infants with PAIS were serially scanned in the neonatal period $(n=15)$, at $3 \mathrm{mo}(n=16)$, and at $24 \mathrm{mo}$ $(n=8)$. Fractional anisotropy (FA) values in five regions of interest (anterior and posterior limb of the internal capsule, corpus callosum, optic radiation, and posterior thalamic radiation) were obtained and compared with FA values of healthy controls and neurodevelopmental outcome.

RESULTS: In the neonatal period, no differences in FA values were found. At $3 \mathrm{mo}$, the six infants who ultimately developed motor deficits showed lower FA values in all affected regions. Four infants developed a visual field defect and showed lower FA values in the affected optic radiation at 3 mo (0.22 vs. 0.29; $P=0.03$ ). Finally, a correlation between FA values of the corpus callosum at $3 \mathrm{mo}$ and the Griffiths developmental quotients was found $(r=0.66 ; P=0.03)$. At $24 \mathrm{mo}$, a similar pattern was observed.

CONCLUSION: Neonatal FA measurements may underestimate the extent of injury following PAIS. FA measurements at 3 mo could be considered a more reliable predictor of neurodevelopmental outcome and correlate with DTI findings at $24 \mathrm{mo}$.

P erinatal arterial ischemic stroke (PAIS), with an incidence of 1 per 2,300-4,000 live births, often leads to adverse neurologic sequelae $(1,2)$. Currently, there are no accepted therapies for the acute treatment of PAIS. However, methods to accurately predict later neurodevelopmental outcome in the first few months following PAIS are rapidly advancing. Magnetic resonance imaging (MRI) plays an important role in the diagnosis of PAIS and in the prognosis of future motor development (3-9).

Several cross-sectional studies have reported differences in diffusion tensor imaging (DTI) parameters following PAIS. Beyond the neonatal period, decreased integrity of the affected corticospinal tracts, reflected by lower fractional anisotropy
(FA) values, is found in children who develop motor deficits $(7,8,10)$. However, no studies have reported longitudinal measurements of DTI following PAIS. In preterm infants, longitudinal studies provide insight into both structural and functional development and have identified possible windows for therapeutic intervention $(11,12)$. The aim of the current study was to examine the potential value of serial DTI following PAIS to evaluate temporal changes in the course of white matter injury and to determine an optimal strategy for prediction of outcome following PAIS.

\section{RESULTS}

\section{Patient Outcome}

The median follow-up duration was 37 mo (range: 19-62 mo). Six children (five with a main trunk middle cerebral artery (MCA) stroke and one with an anterior trunk MCA stroke) developed unilateral motor deficits (UMDs). The median Griffiths developmental quotient was 98 (range: 81-105).

Visual field examinations were performed in 10 children (63\%), including all children with a posterior cerebral artery (PCA) stroke or main trunk MCA stroke. The examinations revealed a visual field defect in four children (three with main trunk MCA stroke and one with PCA stroke). This included a hemianopia in two children and a quadrantanopia in two others.

\section{Region of Interest Analysis and Motor Outcome}

On the first scan, which was acquired during the first week after birth, FA values in the regions of interest (ROIs) in the affected hemisphere did not differ from reference values of the controls. During the first week, median FA values of infants who would later develop UMDs did not differ from those who would not develop UMDs (Table 1). Only two infants with a main trunk MCA stroke, one of whom had an antenatal MCA stroke, had abnormal FA values in several regions in the affected hemisphere on the first scan (Figure 1a). One child with a PCA stroke, who did not develop UMDs, also had abnormal FA values in the posterior thalamic radiation and optic radiation. These abnormal FA values persisted at $3 \mathrm{mo}$.

\footnotetext{
'Department of Neonatology, Wilhelmina Children's Hospital, University Medical Center Utrecht, Utrecht, The Netherlands; ${ }^{2}$ Department of Pediatrics, Eudowood Neonatal Pulmonary Division-NICN, Johns Hopkins University School of Medicine, Baltimore, Maryland; ${ }^{3}$ Department of Neonatology, Children's National Medical Center, Washington, DC; ${ }^{4}$ Department of Ophthalmology, University Medical Center Utrecht, Utrecht, The Netherlands; ${ }^{5}$ Department of Radiology, Johns Hopkins University School of Medicine, Baltimore, Maryland; ${ }^{6}$ F.M. Kirby Functional Imaging Center, Kennedy Krieger Institute, Baltimore, Maryland. Correspondence: Frances J. Northington (frances@jhmi.edu) 
Table 1. Differences in FA values at three time points

\begin{tabular}{llll}
\hline & Neonatal DTI $(n=15)$ & 3 mo DTI $(n=16)$ & 24 mo DTI $(n=8)$ \\
\hline CC & 0.27 vs. $0.28(P=0.55)$ & 0.29 vs. $0.35(P=0.03)$ & 0.37 vs. $0.44(P=0.10)$ \\
PLIC & 0.37 vs. $0.38(P=0.49)$ & 0.40 vs. $0.48(P=0.01)$ & 0.46 vs. $0.55(P=0.10)$ \\
ALIC & 0.20 vs. $0.23(P=0.55)$ & 0.23 vs. $0.29(P=0.02)$ & 0.29 vs. $0.36(P=0.41)$ \\
OR & 0.22 vs. $0.23(P=1.0)$ & 0.22 vs. $0.31(P=0.05)$ & 0.30 vs. $0.37(P=0.10)$ \\
PTR & 0.22 vs. $0.23(P=1.0)$ & 0.22 vs. $0.32(P=0.04)$ & 0.31 vs. $0.37(P=0.41)$ \\
\hline
\end{tabular}

Median fractional anisotropy (FA) values of the five regions of interest-corpus callosum (CC), posterior limb of the internal capsule (PLIC), anterior limb of the internal capsule (ALIC), optic radiation (OR), and posterior thalamic radiation (PTR) — as measured in infants who developed motor deficits vs. those who did not. In the neonatal period, no significant differences were found between the two groups. At 3 mo, significant differences were observed in all regions of interest. These differences could still be observed at 24 mo but no longer reached significance owing to the smaller group of children scanned at 24 mo.

DTI, diffusion tensor imaging.

At $3 \mathrm{mo}$, FA values in the affected hemisphere of infants who later developed UMDs began to clearly deviate from the reference values of controls in several ROIs. The FA values in all affected ROIs of these infants were lower than the corresponding FA values of infants who did not develop UMDs.

In the eight children who were scanned for a third time at $\sim 24$ mo, FA values that were below the $95 \%$ prediction interval at 3 mo remained low at 24 mo (Figure 1b). FA values in the ROIs in the unaffected hemisphere of the children who developed UMDs did not differ from the FA values in the corresponding ROIs of the children who did not develop UMDs at any time point, and FA values in both groups followed the reference curve for the respective regions.

\section{ROI Analysis and Visual Fields}

At 3 mo, FA values of the affected optic radiation were lower in the infants who would later develop a visual field defect $(0.22$ vs. $0.29 ; P=0.03$ ) and were below the reference curve. In three of these infants, FA values were also abnormal on the neonatal scan (Figure 2). Of the six children with normal visual fields, five had normal FA values in the affected optic radiation at 3 mo. One child with a main trunk MCA stroke had an abnormal FA value in the affected optic radiation at 3 mo without a detectable visual field defect.

No visual field test was acquired in the six remaining children. There was, however, no history suggestive of a visual field defect in these children, and the FA values of their affected optic radiations were within the $95 \%$ prediction intervals at 3 mo.

A correlation was observed between the Griffiths developmental quotient and the FA of the corpus callosum on the second scan $(r=0.66 ; P=0.03$; Figure 3$)$. No correlation between the Griffiths developmental quotient and FA values of other ROIs was found at any time.

\section{Maps of Standard Z Scores}

The $Z$ maps showed findings similar to those of the ROI analysis. In an infant with a main trunk MCA stroke (Figure 4), the neonatal scan showed no apparent abnormality in FA values; however, the 3-mo scan clearly indicated decreased FA in the posterior limb of the internal capsule and several other white matter tracts. This spatial pattern of abnormalities largely persisted on the 24-mo scan.
The $Z$ maps provide additional information, revealing potential local abnormalities not detected with ROI analysis. For example, the low FA value in the splenium of the corpus callosum in an infant with a PCA stroke (Supplementary Figure S1 online) was not detected by examination of the FA value of the corpus callosum ROI.

\section{DISCUSSION}

In the current study, serial DTI data of infants diagnosed with PAIS were acquired to examine the temporal evolution of white matter injury and correlate this with different outcome domains. We were able to demonstrate time-related changes in FA values in regions commonly affected by PAIS. Although neonatal FA values tended to underestimate injury, FA values at 3 mo more accurately showed the full extent of injury and correlated with FA values at $24 \mathrm{mo}$ and with long-term neurodevelopmental outcome.

Little is known about the changes in FA during the first week following PAIS. Both increased and decreased FA values have been reported following hypoxic-ischemic injury $(13,14)$. In adult stroke, an initial increase in FA values is followed by a decrease from day 5 onward (15). The initial increase is thought to reflect reduced spacing between the axons owing to the cytotoxic edema, whereas the subsequent decrease in FA reflects loss of structural integrity. This suggests that the timing of FA measurements following stroke is important, as observed in the infant with antenatal stroke in this study. On the basis of different MRI sequences, this infant most likely suffered a stroke 10-14 d before birth, and this could explain why abnormal FA values were already present on the neonatal scan. Similar abnormally low FA values were not observed in the other infants with neonatal stroke who would later develop UMDs.

At 3 mo, clear differences in FA values were observed, and these differences corresponded with development of motor deficits, cognitive outcome, and development of visual field defects. Even though this would suggest that 3 mo might be a better time point for predicting outcome using FA measurements, DTI at this time cannot replace neonatal MRI. During the neonatal period, conventional MRI and diffusion-weighted imaging remain essential for the diagnosis of PAIS and have also shown their value in predicting the development of motor disabilities $(3,4,6,9)$. However, conventional MRI and diffusion-weighted imaging cannot be used to quantify the severity of white matter injury related to the 
a
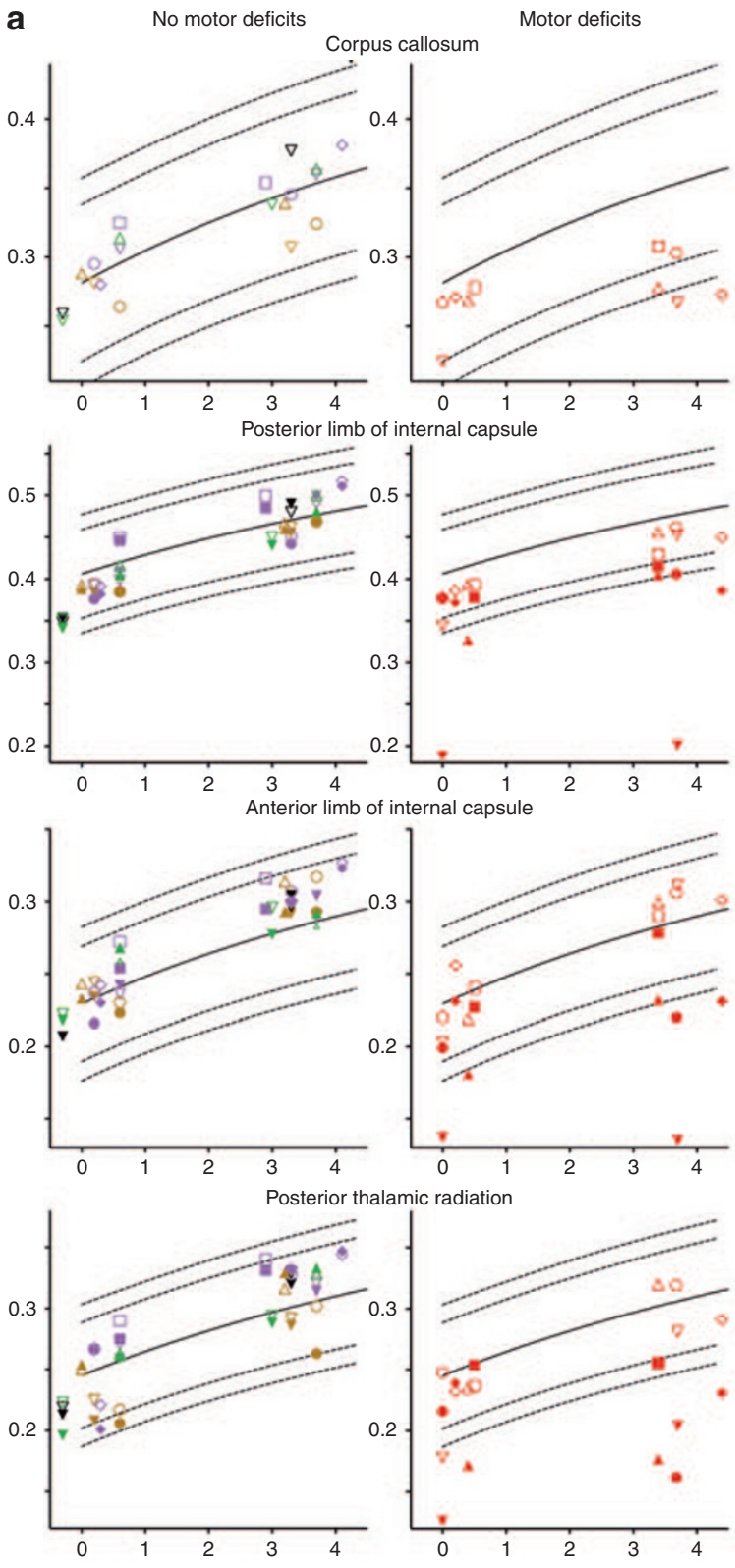

Optic radiation

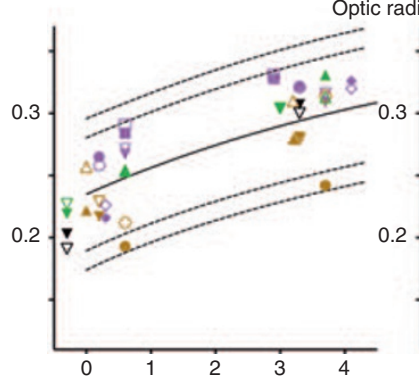

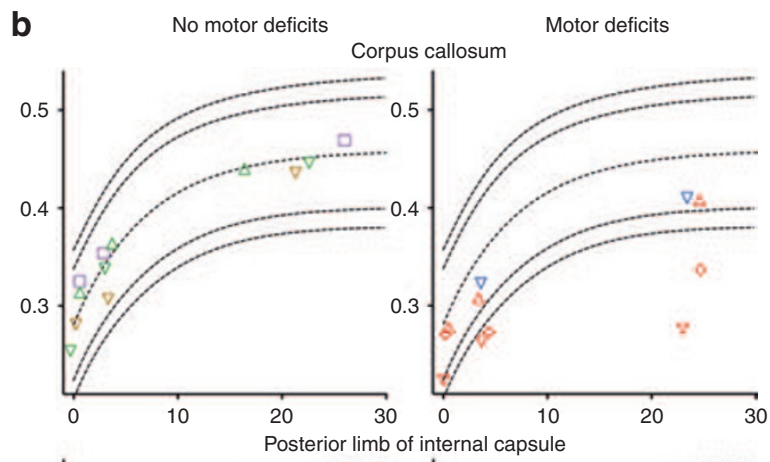
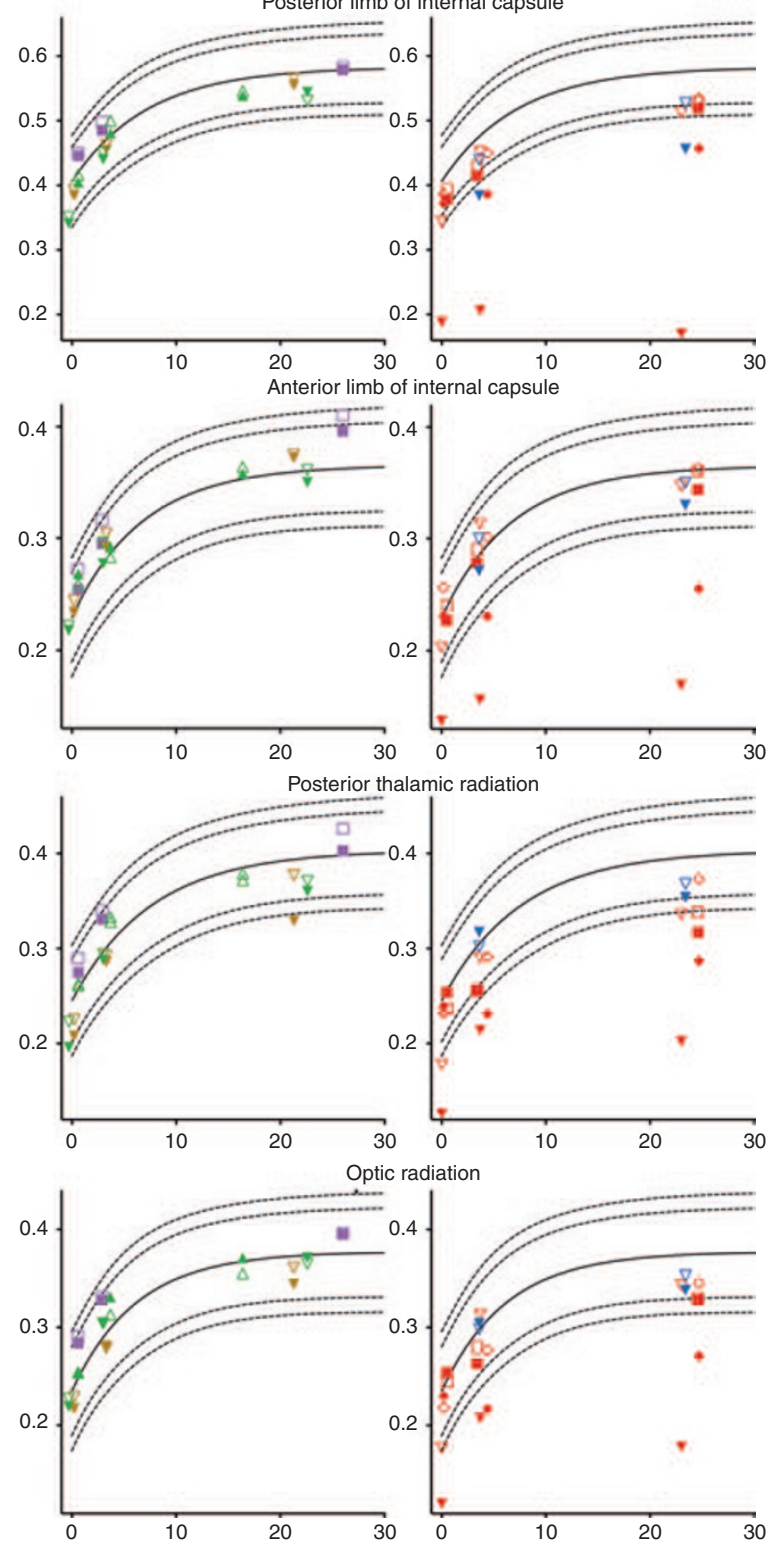

Figure 1. The fractional anisotropy (FA) values (vertical axis) in the different regions of interest (ROIs) vs. the age at scan (horizontal axis) of (a) 16 infants who were scanned in the neonatal period and at 3 mo and of the (b) 8 infants who were also scanned at 24 mo, FA values are shown for infants with normal motor development (left column) and those with motor deficits (right column). The FA values of the affected side are depicted by closed symbols, whereas the FA values of the unaffected side are depicted by the corresponding open symbols. For the corpus callosum, the mean FA was determined and depicted. The different colors represent the different kinds of strokes observed: main branch middle cerebral artery (MCA) stroke (red), anterior branch MCA stroke (blue), posterior branch MCA stroke (green), cortical MCA branch (purple), lenticulostriate branch MCA stroke (black), and posterior cerebral artery stroke (brown). The child with the antenatal MCA stroke is depicted as an upside-down triangle. The dashed lines represent the 95 and $99 \%$ prediction intervals for each ROI. 


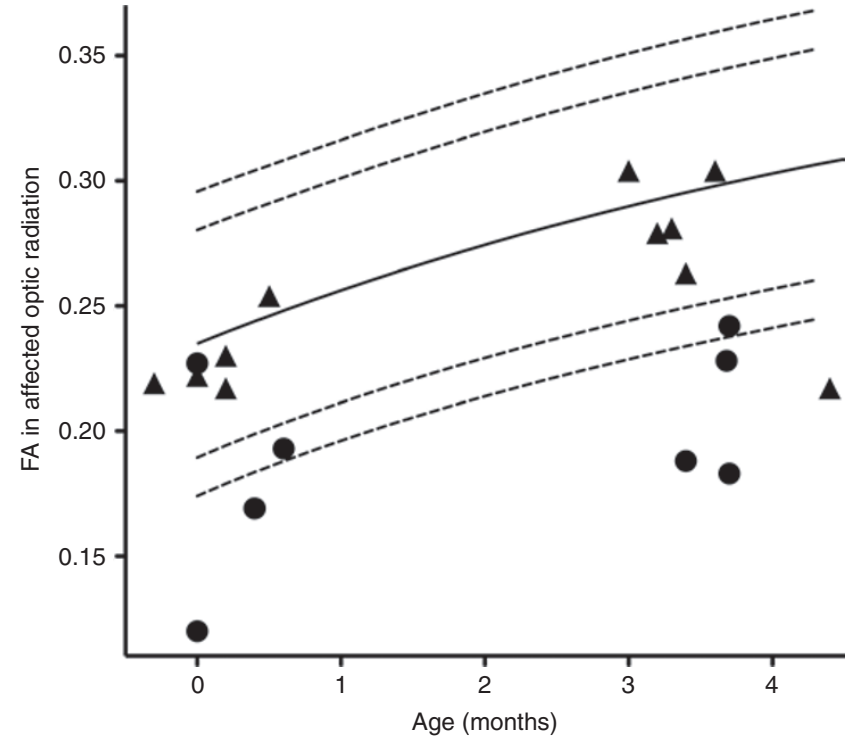

Figure 2. Fractional anisotropy (FA) values of the affected optic radiation in 10 infants subsequently tested for visual field defects. Circles represent infants who developed a visual field defect, whereas triangles represent infants with a normal visual field examination on follow-up. The dashed lines represent the 95 and $99 \%$ prediction intervals for each region of interest.

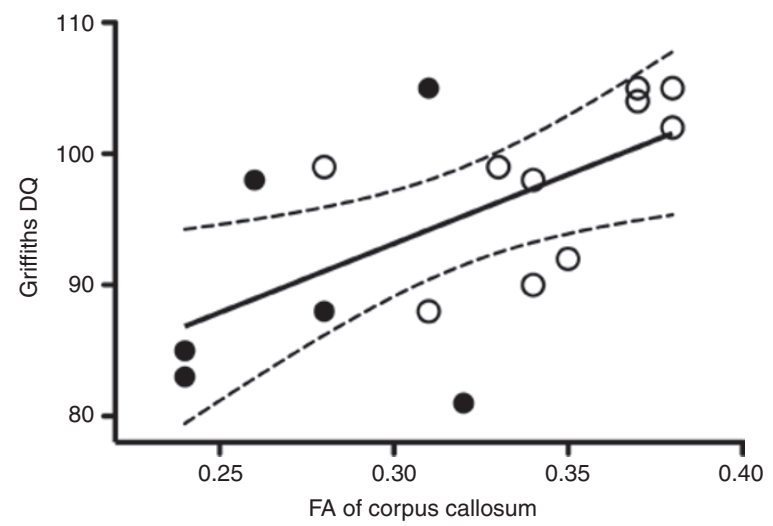

Figure 3. Correlation between the fractional anisotropy (FA) value of the corpus callosum at the age of $3 \mathrm{mo}$ and the Griffiths developmental quotient (DQ) score (24) at later follow-up, displayed as a regression line (continuous line) and its 95\% confidence interval (dashed line). The infants who developed unilateral motor deficits are displayed as filled symbols.

development of motor disabilities or visual field defects, as can be done using DTI, even before the development of clinical symptoms $(8,10)$. We therefore propose that DTI should be used after the acute phase of injury, i.e., after $4-6 \mathrm{wk}$, to evaluate the extent of injury and assist with the prediction of neurodevelopmental outcome other than UMDs in at-risk infants.

Visual field defects following PAIS occur in $20 \%$ of all cases $(9,16)$. Prediction of visual field defects using conventional MRI is difficult as involvement of the optic radiation or visual cortex does not always correlate with deficits. In preterm infants, DTIbased tractography of the optic radiation at term-equivalent age is associated with visual function (17). Although the number of children who underwent a visual field exam in this study was limited to those with lesions known to be associated with visual

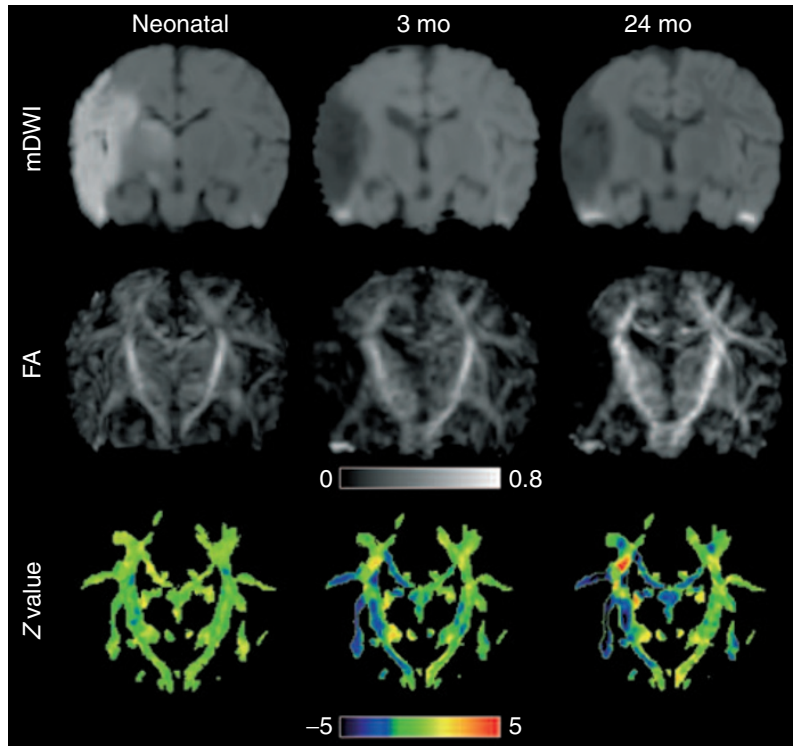

Figure 4. Coronal views of serial diffusion tensor imaging data sets after normalization to the template. The neonatal mean diffusion-weighted image $(\mathrm{mDWl})$ shows an ischemic area in the middle cerebral artery territory, which resulted in a cyst observed at 3 and $24 \mathrm{mo}$. The neonatal $Z$ map shows normal $Z$ values in all regions. At 3 mo, low $Z$ values, representing abnormal fractional anisotropy (FA) values, were observed in the affected corticospinal tract, which corresponded with the subsequent development of unilateral motor deficits. The $Z$ map at 24 mo was essentially identical. Interestingly, FA values in the fornix continued to decrease from the time of the neonatal scan to $24 \mathrm{mo}$, suggesting delayed degeneration in this tract.

field deficits, abnormalities in FA values of the optic radiation at 3 mo predicted visual field defects. Larger studies will be needed to confirm this finding and may allow differentiation between development of a hemianopia and quadrantanopia.

Using the $Z$ maps, we examined the spatial pattern of white matter injury in both the acute and postacute phase. This method allowed visualization of white matter integrity over time without predefined ROIs. Both preterm and term studies have shown that brain injury can result in widespread abnormalities of white matter development at sites remote from a focal lesion $(18,19)$. This was also observed in the current study, e.g., in the corpus callosum and fornix (4). The importance of such widespread white matter injury is well known from preterm infants who may show multiple defects in brain development, including delayed or abnormal myelination, abnormal cortical folding, and decreased thalamic volume associated with global white matter injury (19), and these abnormalities almost certainly underlie the complex neurobehavioral deficits found in these children. It is reasonable to expect that similar conclusions can be drawn from our observations made with regard to white matter integrity in full-term infants with PAIS.

\section{Technical Considerations}

One should note that the control and patient images in this study were acquired using different scanners with different settings, which may have introduced a scanner-dependent bias (20). Assessing such bias would require careful calibration of scanner instruments. Although we were not able to do this, we 

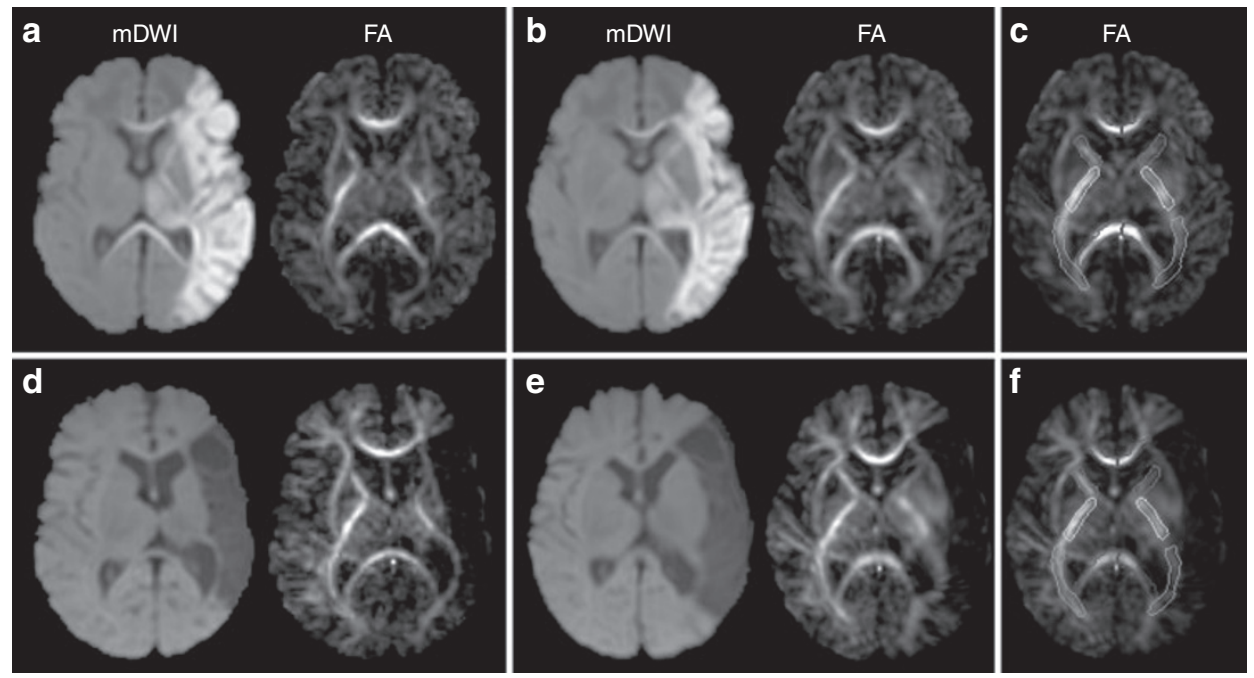

Figure 5. Example of the normalization of two diffusion tensor imaging data sets acquired in the (a-c) neonatal period and (d-f) at the age of 3 mo in an infant with a stroke in the main trunk of the middle cerebral artery. (a,d) After the affine registration and $(\mathbf{b}, \mathbf{e})$ subsequent LDDMM procedure, (c,f) FA values were determined in the regions of interest. FA, fractional anisotropy; LDDMM, large deformation diffeomorphic metric mapping; mDWI, mean diffusion-weighted image.

were able to show that the FA values determined in the ROIs in the unaffected hemisphere followed the reference curve, suggesting that if any scanner-dependent bias in FA values was present, it was minimal.

The reported results strongly depend on an accurate normalization of the data to the atlas. Although large deformation diffeomorphic metric mapping (LDDMM) has been used before in older children with cerebral palsy (21), to our knowledge this paper is the first attempt to normalize images from infants with severe injury, e.g., the presence of a large ischemic lesion or cyst. For these cases, time-consuming landmark-based normalization, expert knowledge, and careful inspection were necessary for accurate normalization. This procedure is therefore not yet suitable for daily clinical practice. Incorporation of automatic lesion segmentation techniques and masking of these regions during the registration process may allow easier access to the clinician $(22,23)$.

Limitations of the study were the relatively small sample size and the absence of a visual field test in six children. The data in this study were collected retrospectively, and visual field tests were therefore only administered when clinically indicated.

\section{Conclusion}

Serial DTI following PAIS allows insight into the evolution of white matter injury following PAIS. Although neonatal DTI may not capture the full extent of the injury, DTI at 3 mo does, and it corresponds well with the DTI findings at $24 \mathrm{mo}$ in the major white matter tracts examined and with long-term motor, cognitive, and visual outcomes.

\section{METHODS}

\section{Patient Selection and Neurodevelopmental Assessment}

This retrospective study included 16 full-term infants $(11$ male; median gestational age $40 \mathrm{wk}$; range: $37^{2 / 7}-41^{3 / 7}$ ), who were cared for in the neonatal intensive care unit at the Wilhelmina Children's
Hospital (Utrecht, The Netherlands), were diagnosed with PAIS, and underwent cranial MRI during the first week after birth. DTI data were acquired during the neonatal period in 15 of the infants. Strokes were observed in the territories of the PCA $(n=3)$ and MCA $(n=13)$. One infant's MRI showed a main trunk MCA stroke on T2, with little diffusion restriction, suggesting antenatal onset of the stroke, $\sim 10-14$ d before birth.

No permission was required from the institutional review board for this study. Informed parental consent was obtained before each MRI scan. Eleven of the included infants were studied as part of a larger cohort, in which DTI-based tractography of the corticospinal tracts was performed at the age of $3 \mathrm{mo}$, as reported previously (8).

A second MRI was obtained at a median age of 3.4 mo (range: 2.9-4.4 mo) in all children, and eight children were scanned again at a median age of $23.2 \mathrm{mo}$ (range: 16.4-29.6 mo). Neurodevelopmental outcome was evaluated between 18 and 36 mo using the Griffiths mental developmental scale (24). The presence of UMDs was assessed according to Claeys (25).

In infants in whom the development of a visual field defect was thought to be likely owing to the involvement of the optic radiation, cortex, or thalamus, an experienced pediatric ophthalmologist performed visual field examinations. This was feasible from the age of 3 mo onward using the behavioral visual field screening test (BEFIE test) (26). In this test, the examiner stands behind the child and rotates an arc with a ball attached to its tip around the child's head, from the periphery to the center of the visual field. The angle at which the child first detects the stimulus determines the border of the visual field. Visual fields were scored as normal, presence of a quadrantanopia, or presence of a hemianopia.

\section{DTI Protocol}

Patients were scanned on a 1.5T Philips MR scanner (Philips Medical Systems, Best, The Netherlands) using a SENSE head coil. The neonatal and 3-mo DTI protocol consisted of a single-shot echo-planar imaging sequence (echo-planar imaging factor: 43; repetition time/ echo time: $6,817 / 87 \mathrm{~ms}$ ) with 50 axial $2 \mathrm{~mm}$ slices in 32 noncollinear diffusion-weighted directions with a $b$ value of $800 \mathrm{~s} / \mathrm{mm}^{2}$ and one $b=0 \mathrm{~s} / \mathrm{mm}^{2}$ image (field of view: $190 \times 190 \mathrm{~mm}$; acquisition matrix: $96 \times 96$; reconstruction matrix: $128 \times 128$ ). At $2 \mathrm{y}$, a similar single-shot echo-planar imaging sequence was used but with an echo-planar imaging factor of 51, a repetition time of $8,382 \mathrm{~ms}$, and field of view of $192 \times 192 \mathrm{~mm}$. 
During the first two scans, infants were sedated as described previously (8). A vacuum pillow (Med-Tec, Orange City, IA) was used to prevent head movement. Minimuffs (Natus Medical, San Carlos, CA) were used for hearing protection, and a neonatologist was present throughout the examination. At $2 \mathrm{y}$, children received general anesthesia, were ventilated, and the heart rate, ventilation, and transcutaneous oxygen saturation were monitored by an anesthetist.

\section{Control Subjects}

DTI scans of 42 healthy children (age: 0-30 mo) were used to create reference curves for the different ROIs and to create age-matched templates. These data were obtained from a database of normative scans, which were acquired on a $1.5 \mathrm{~T}$ Philips MR scanner at Johns Hopkins University (27).

\section{Image Post-Processing}

Images were processed with ExploreDTI (http://www.exploredti. com). The diffusion-weighted images were realigned to the b0 image to correct for subject motion and eddy current-induced geometric distortions. In this process, the diffusion tensor was fitted for each voxel after adjusting the diffusion gradients for the b-matrix rotation (28). After this procedure, the mean diffusion-weighted image (mDWI), the b0 image, and the FA image were exported for further analysis. Finally, the brain volume was extracted manually by removing signals in the mDWI images from tissues outside the brain (skull stripping).

Furthermore, the DTI data were normalized to a single-subject neonatal DTI template, consisting of an $\mathrm{mDWI}, \mathrm{FA}$, and b0 image, which are freely available (http://cmrm.med.jhmi.edu). The mDWI image of each subject was aligned with the mDWI of the template using a 12-mode affine transformation provided by the automated image registration software (http://loni.ucla.edu/Software/AIR) (Figure 5) (29). This transformation was then applied to the FA images and followed by dual-contrast (FA and $\mathrm{mDWI}$ ) LDDMM as provided by the Diffeomap software (https://www.mristudio.org), using descending a values of $0.05,0.04$, and 0.03 to achieve coarse-to-fine normalization $(30,31)$. In infants with a large MCA stroke, the extensive high signal intensity on the mDWI and the subsequent cyst at 3 mo hindered accurate normalization. In these infants, the LDDMM at 0 mo was driven by the $\mathrm{b} 0$ and FA images. At 3 mo, the LDDMM was preceded by a landmark-based LDDMM with 180-200 landmarks to bring structures displaced by the cyst close to their normal locations as in the template (32). The result was further improved by a single $\alpha(0.05)$ dual-contrast LDDMM and visually inspected to ensure accurate normalization for the major white matter tracts.

\section{Region of Interest-Based Analysis}

After normalization of the controls to the template, FA values were determined in the normalized FA images using five ROIs defined in the template image. These ROIs included the corpus callosum, the posterior and anterior limb of the internal capsule, the posterior thalamic radiation, and the optic radiation. This procedure produced consistent definitions of ROIs among subjects. FA values of bilateral ROIs were averaged for each control and plotted against age. GraphPad Prism v5.0 (GraphPad Software, San Diego, CA) was used to fit FA values as a function of age of the controls, using an exponential regression model. The 95 and 99\% prediction intervals were calculated for each ROI separately (Supplementary Figure S2 online). FA values in these five ROIs were also determined for the patients and were plotted in the curves of the controls. FA values of patients were regarded normal when they fell within the $95 \%$ prediction intervals and abnormal if they fell below the intervals.

\section{Maps of Standard Z Scores}

For each voxel in the template with an FA value $>0.2$, an exponential fit was performed, fitting the FA values of the controls as a function of age using Matlab R2011a (Mathworks, Natick, MA). The mean FA value and the standard error of each voxel were estimated at different ages from the exponential fitting and were used to calculate standard $Z$ scores with respect to the controls for each voxel as:

$$
Z=\frac{X_{\text {patient }}-\bar{X}_{\text {control }}}{\mathrm{SE}}
$$

where $X_{\text {patient }}$ is the FA value of the patient's voxel and $\bar{X}_{\text {control }}$ and SE are the mean FA and standard error of the control subjects at the voxel.

\section{Statistical Analyses}

Differences in clinical parameters or FA values between groups were tested using the Mann-Whitney $U$ test. The Bonferonni post hoc test was used for testing multiple ROIs at each time point. Spearman's correlation coefficient was used to determine the relation between the Griffiths developmental quotient and FA values.

\section{SUPPLEMENTARY MATERIAL}

Supplementary material is linked to the online version of the paper at http:// www.nature.com/pr

\section{STATEMENT OF FINANCIAL SUPPORT}

N.E.v.d.A. was supported by grants from the Ter Meulen Foundation, Foundation 'De Drie Lichten', the Wilhelmina Children's Research Foundation, and the Dutch Phelps Foundation for this project. Further financial support was received from the March of Dimes Foundation (6-08-275 to F.J.N.) and the National Institutes of Health (NS 059529 to J.Z.).

Disclosure: The authors declared no conflict of interest.

\section{REFERENCES}

1. Nelson KB, Lynch JK. Stroke in newborn infants. Lancet Neurol 2004;3:150-8.

2. Kirton A, deVeber G. Advances in perinatal ischemic stroke. Pediatr Neurol 2009;40:205-14.

3. Kirton A, Shroff M, Visvanathan T, deVeber G. Quantified corticospinal tract diffusion restriction predicts neonatal stroke outcome. Stroke 2007;38:974-80.

4. De Vries LS, Van der Grond J, Van Haastert IC, Groenendaal F. Prediction of outcome in new-born infants with arterial ischaemic stroke using diffusionweighted magnetic resonance imaging. Neuropediatrics 2005;36:12-20.

5. Lee J, Croen LA, Lindan C, et al. Predictors of outcome in perinatal arterial stroke: a population-based study. Ann Neurol 2005;58:303-8.

6. Husson B, Hertz-Pannier L, Renaud C, et al.; AVCnn Group. Motor outcomes after neonatal arterial ischemic stroke related to early MRI data in a prospective study. Pediatrics 2010;126:912-8.

7. Roze E, Harris PA, Ball G, et al. Tractography of the corticospinal tracts in infants with focal perinatal injury: comparison with normal controls and to motor development. Neuroradiology 2012;54:507-16.

8. van der Aa NE, Leemans A, Northington FJ, et al. Does diffusion tensor imaging-based tractography at 3 months of age contribute to the prediction of motor outcome after perinatal arterial ischemic stroke? Stroke 2011;42:3410-4.

9. Mercuri E, Anker S, Guzzetta A, et al. Neonatal cerebral infarction and visual function at school age. Arch Dis Child Fetal Neonatal Ed 2003;88:F487-91.

10. Glenn OA, Ludeman NA, Berman JI, et al. Diffusion tensor MR imaging tractography of the pyramidal tracts correlates with clinical motor function in children with congenital hemiparesis. AJNR Am J Neuroradiol 2007;28:1796-802.

11. Smyser CD, Inder TE, Shimony JS, et al. Longitudinal analysis of neural network development in preterm infants. Cereb Cortex 2010;20:2852-62.

12. Groppo M, Ricci D, Bassi L, et al. Development of the optic radiations and visual function after premature birth. Cortex 2012 (http://dx.doi. org/10.1016/j.cortex.2012.1002.1008).

13. Ward P, Counsell S, Allsop J, et al. Reduced fractional anisotropy on diffusion tensor magnetic resonance imaging after hypoxic-ischemic encephalopathy. Pediatrics 2006;117:e619-30.

14. van Pul C, Buijs J, Vilanova A, Roos FG, Wijn PF. Infants with perinatal hypoxic ischemia: feasibility of fiber tracking at birth and 3 months. Radiology 2006;240:203-14. 


\section{Articles $\mid$ van der Aa et al.}

15. Sotak $\mathrm{CH}$. The role of diffusion tensor imaging in the evaluation of ischemic brain injury - a review. NMR Biomed 2002;15:561-9.

16. van der Aa NE, Dudink J, Benders MJ, et al. Neonatal posterior cerebral artery stroke: clinical presentation, MRI findings, and outcome. Dev Med Child Neurol 2013;55:283-90.

17. Bassi L, Ricci D, Volzone A, et al. Probabilistic diffusion tractography of the optic radiations and visual function in preterm infants at term equivalent age. Brain 2008;131(Pt 2):573-82.

18. Ramenghi LA, Rutherford M, Fumagalli M, et al. Neonatal neuroimaging: going beyond the pictures. Early Hum Dev 2009;85:Suppl 10:S75-7.

19. Ment LR, Hirtz D, Hüppi PS. Imaging biomarkers of outcome in the developing preterm brain. Lancet Neurol 2009;8:1042-55.

20. Huisman TA, Loenneker T, Barta G, et al. Quantitative diffusion tensor MR imaging of the brain: field strength related variance of apparent diffusion coefficient (ADC) and fractional anisotropy (FA) scalars. Eur Radiol 2006;16:1651-8.

21. Faria AV, Hoon A, Stashinko E, et al. Quantitative analysis of brain pathology based on MRI and brain atlases - applications for cerebral palsy. Neuroimage 2011;54:1854-61.

22. Brett M, Leff AP, Rorden C, Ashburner J. Spatial normalization of brain images with focal lesions using cost function masking. Neuroimage 2001;14:486-500.

23. Ghosh N, Yuan X, Turenius CI, et al. Automated core-penumbra quantification in neonatal ischemic brain injury. J Cereb Blood Flow Metab 2012;32:2161-70.
24. Griffiths R. The Abilities of Babies: A Study in Mental Measurement. Amersham, United Kingdom: Association for Research in Infants and Child Development, 1976.

25. Claeys V, Deonna T, Chrzanowski R. Congenital hemiparesis: the spectrum of lesions. A clinical and computerized tomographic study of 37 cases. Helv Paediatr Acta 1983;38:439-55.

26. Porro GJ, Hofmann J, Wittebol-Post D, et al. A new behavioral visual field test for clinical use in pediatric neuro-ophthalmology. Neuro-opthalmology 1998;19:205-14.

27. Hermoye L, Saint-Martin C, Cosnard G, et al. Pediatric diffusion tensor imaging: normal database and observation of the white matter maturation in early childhood. Neuroimage 2006;29:493-504.

28. Leemans A, Jones DK. The B-matrix must be rotated when correcting for subject motion in DTI data. Magn Reson Med 2009;61:1336-49.

29. Woods RP, Grafton ST, Holmes CJ, Cherry SR, Mazziotta JC. Automated image registration: I. General methods and intrasubject, intramodality validation. J Comput Assist Tomogr 1998;22:139-52.

30. Miller MI, Trouve A, Younes L. On the metrics and euler-lagrange equations of computational anatomy. Annu Rev Biomed Eng 2002;4: 375-405.

31. Ceritoglu C, Oishi K, Li X, et al. Multi-contrast large deformation diffeomorphic metric mapping for diffusion tensor imaging. Neuroimage 2009; 47:618-27.

32. Joshi SC, Miller MI. Landmark matching via large deformation diffeomorphisms. IEEE Trans Image Process 2000;9:1357-70. 